\title{
Outpatient total hip arthroplasty : the future?
}

\author{
Solange De Wouters, Steven Petronilia, Daniel Paulet, Tom De Baere, Etienne Willemart, Olivier Cornu
}

From the Saint-Jean Hospital, Brussels, Belgium

With advancements in minimally invasive surgical technique, pain management and rehabilitation protocols, and prevention of post-operative complications, outpatient total hip arthroplasty became a realistic goal. This study reports our experience of performing outpatient total hip arthroplasty assessing its feasibility and safety.

Between December 2015 and January 2018, 52 outpatient total hip arthroplasties were performed. We implemented a peri-operative management protocol that included education, improved analgesia and rapid rehabilitation. Patients were asked about any complications they had experienced after surgery and about the perception of their experience as outpatient. Any early post-operative emergency department visits, acute office appointments or hospital re-admissions were registered.

Fifty-one of 52 patients $(98,1 \%)$ enrolled in the study met the discharge criteria and achieved their goal of going home on the day of surgery, and only one patient $(1,9 \%)$ required an overnight stay. There were two visits to the emergency room, with one hospital re-admission $(1,9 \%)$ on the night of the surgery. There were no major post-discharge complications in the short-term follow-up (minimum of three months). Only one patient $(1,9 \%)$ reported a significantly negative experience.

With this first Belgian experience reporting on outpatient primary total hip arthroplasty, our data demonstrate that early discharge does not result in excessive re-admissions or other post-discharge complications due to an early discharge. This study suggests that total hip arthroplasty can be performed safely and successfully in appropriately selected patients and that patients who experienced that

No benefits or funds were received in support of this study. None of the authors have a conflict of interest. ambulatory protocol declared themselves highly satisfied.

Keywords : total hip arthroplasty ; outpatient ; ambulatory ; safety.

\section{INTRODUCTION}

Nowadays, there is a clear trend worldwide towards early discharge of patients from hospital, as soon as this is safely possible. Two elements led to earlier hospital discharge after primary THA : the increased demand in a younger and healthy population for THA (1) and the implementation of

Solange De Wouters ${ }^{1,2}, \mathrm{MD}$,

- Steven Petronilia ${ }^{1,3}, \mathrm{MD}$,

Daniel Paulet ${ }^{1,3}, \mathrm{MD}$,

Tom De Baere ${ }^{1,3}, \mathrm{MD}$,

Etienne Willemart ${ }^{1,3}, \mathrm{MD}$

- Olivier Cornu ${ }^{1,2}, \mathrm{PhD}$

${ }^{1}$ Department of Orthopaedic Surgery, Clinique Saint-Jean, Boulevard du Jardin Botanique 32, 1000 Brussels, Belgium. ${ }^{2}$ Department of Orthopaedic Surgery, Cliniques universitaires Saint-Luc, Avenue Hippocrate 10, 1200 Brussels, Belgium.

${ }^{3}$ Department of Orthopaedic Surgery, CHIREC - Hôpital Delta, Boulevard du Triomphe 201, 1160 Brussels, Belgium.

Correspondence : Solange De Wouters, MD, Department of Orthopaedic Surgery, Clinique Saint-Jean, Boulevard du Jardin Botanique 32, 1000 Brussels, Belgium.

Email : solange.dewouters@uclouvain.be

- 2021, Acta Orthopædica Belgica. 
fast-track protocols (2). Furthermore, we cannot ignore the economic pressure on reducing healthcare costs, nor the patients' desire for a rapid recovery enabling them to enjoy the immediate benefits of THA in their own home environment. Thereby, the transition from an inpatient to an outpatient THA practice occurred as a natural progression. With advancements in minimally invasive surgical technique, improved pain management, new rehabilitation protocols and prevention of post-operative complications, outpatient THA became a realistic goal in selected patients.

This study reports our experience of performing outpatient THA using an integrated anesthesia and orthopedic protocol for 52 THAs in a group of 49 selected patients. We assessed the feasibility, safety and clinical outcome of outpatient THA and we tried to identify the common peri-operative challenges encountered with a same-day discharge. The purpose of this paper is to help assessing whether a same-day discharge would be beneficial for the patients who choose it.

\section{MATERIALS AND METHODS}

To successfully achieve THA on an outpatient basis, clinicians have developed accelerated clinical pathways (3). We implemented a perioperative management protocol that included intensive pre-operative education, intra-operative anesthetic technique, improved post-operative analgesia, meticulous monitoring for post-operative complications and rehabilitation protocols with rapid mobilization that allow an early discharge. The goal was to allow safe home discharge from the hospital on the day of the surgery, at late afternoon.

We designed a prospective cohort study to offer a same-day program for all patients younger than 75 years who wished to do so. Between December 2015 and January 2018, 52 outpatient THAs were performed at a single institution. All operations were performed by the same orthopedic surgeon (EW).

All patients who were clinically and radiographically indicated for primary THA (having failed conservative treatment) and considered sufficiently healthy for early discharge were given the option of outpatient THA. Patients with cardio-pulmonary abnormality, cirrhosis, insulin-requiring diabetes or neurological disease were excluded, as well as patients taking anticoagulants. The selection of patients was then based on the American Society of Anesthesiologists (ASA) physical status classification, a validated score of patient health (4): in theory, patients with an ASA higher than II were not candidates for outpatient THA. Theoretically, the study was limited to patients younger than 75 years of age and a body mass index (BMI) of $35 \mathrm{~kg} / \mathrm{m}^{2}$ was used as the cutoff point for being a candidate. However, if patients (with ASA III, older than 75 years or with a BMI greater than 35) were otherwise healthy and were deemed to have a body habitus that would not interfere with surgery, rarely could be indicated for outpatient THA. Furthermore, family (or friend) support was necessary for inclusion in this study, to be present at home with the patient during the first 72 hours post-operatively. Patients needed to live at less than $20 \mathrm{~km}$ from any hospital. Patients needed to be motivated to participate and all patients who chose to enroll in this study, signed their informed consent before participating, as did their general practitioner.

Pre-operatively, all patients and their family support were invited to attend an educational class of recovery. Each patient also met a physiotherapist trained by our team to review post-operative early mobilisation. Finally, the patient met the anesthesiologist to confirm that he/she was an appropriate candidate for outpatient THA and to discuss the pain management protocol.

All patients were admitted on the day of surgery, 90 minutes before the scheduled surgical procedure. The protocol called for the outpatient THA to be performed as the first case of the day, starting at 8 am. All patients had THA performed with a minimally invasive direct anterior approach, on a standard operating table. All patients had a hemispheric, porous-coated acetabular cup (Pinnacle ${ }^{\circledR}$, Depuy) and a full porous-coated stem (Corail ${ }^{\circledR}$, Depuy) with a ceramic-on-ceramic interface. Prostheses were uncemented on both the acetabular and femoral sides.

Prophylactic intravenous (IV) antibiotics (2 g of Cefazoline ${ }^{\circledR}$ ) were administered 15 minutes prior 
to skin incision. All patients received $1 \mathrm{~g}$ of IV Exacyl ${ }^{\circledR}$ at induction. Patients had no compression stockings.

One week before surgery, patients had to take $5 \mathrm{mg}$ of OxyContin ${ }^{\circledR}$ as a trial run in order to test for potential secondary effects. If side effects occurred, OxyContin ${ }^{\circledR}$ was replaced with $100 \mathrm{mg}$ of Contramal ${ }^{\circledR}$.

All patients were administered a general endotracheal anesthesia for the entire surgery. Diprivan ${ }^{\circledR}$ was titrated intra-operatively for sedation. Perioperatively, patients also received $4 \mathrm{mg}$ of Ondansetron ${ }^{\circledR}$ to decrease post-operative nausea. Thirty milligrams of Taradyl ${ }^{\circledR}$ and Aacidexam ${ }^{\circledR}(0,1 \mathrm{mg} /$ $\mathrm{kg}$ ) were given intra-operatively for improved pain control. Just before the wound closure, we performed a periarticular anesthetic infiltration consisting of Adrenaline ${ }^{\circledR}$ and Naropin ${ }^{\circledR}$. No patients received nerve block.

Peri-operative anesthesia was performed using a multimodal pain management protocol that included medications provided pre-operatively and post-operatively (Table I).

Patients were evaluated in the recovery room by the surgeon and physiotherapy was initiated as tolerated within two hours of termination of surgery for an immediate weight bearing with crutches. Patients received a second physiotherapy session at $1 \mathrm{pm}$ for practice walking using crutches, and a third session at $3 \mathrm{pm}$ for stairs.

If patients experienced any difficulties with nausea, pain or orthostatic hypotension, one of the clinical nurses was called immediately to respond.

All patients were examined carefully on the hospital ward at $2 \mathrm{pm}$ by the surgeon and the anesthesiologist for any intra-operative or early post-operative complications. A final dose of IV antibiotics was administered 8 hours after the first dose. No additional antibiotics were given while patients were at home. All patients received an injection of $40 \mathrm{mg}$ of Clexane ${ }^{\circledR}$ once a day as deep venous thrombosis prophylaxis for 30 days, with the initial dose being given at $6 \mathrm{pm}$ the day of the surgery. Ice was applied to the affected hip for a period of 20 minutes three times during the day. Anteroposterior and lateral radiographs were obtained on the floor.
Table I - Multimodal pain protocol

\begin{tabular}{|c|c|}
\hline Medications & Dose \\
\hline \multicolumn{2}{|l|}{ Preoperative } \\
\hline \multicolumn{2}{|l|}{2 hours before surgery: } \\
\hline OxyContin $\AA$ & $10 \mathrm{mg}$ PO \\
\hline Dafalgan $\AA$ Forte & $1000 \mathrm{mg}$ PO \\
\hline \multicolumn{2}{|l|}{ Postoperative } \\
\hline \multicolumn{2}{|l|}{4 hours after surgery: } \\
\hline Dafalgan $®$ Forte & $1000 \mathrm{mg}$ IV \\
\hline \multicolumn{2}{|l|}{8 hours after surgery: } \\
\hline Taradyl® & $30 \mathrm{mg}$ IV \\
\hline \multicolumn{2}{|l|}{10 hours after surgery: } \\
\hline Dafalgan ${ }^{\circledR}$ Forte & $1000 \mathrm{mg}$ IV \\
\hline \multicolumn{2}{|l|}{12 hours after surgery: } \\
\hline OxyContin $\AA$ & $10 \mathrm{mg}$ PO \\
\hline \multicolumn{2}{|l|}{14 hours after surgery: } \\
\hline Vimovo® & $500 \mathrm{mg}$ PO \\
\hline \multicolumn{2}{|l|}{16 hours after surgery: } \\
\hline Dafalgan $₫$ Forte & $1000 \mathrm{mg}$ PO \\
\hline \multicolumn{2}{|l|}{ Rescue medication: } \\
\hline OxyNorm ${ }^{\circledR}$ Instant & $5 \mathrm{mg}$ PO \\
\hline \multicolumn{2}{|l|}{ Day 1} \\
\hline Dafalgan $\AA$ Forte & 1000 mg PO every 6 hours \\
\hline Vimovo $\AA$ & $500 \mathrm{mg}$ PO every 12 hours \\
\hline OxyContin $\mathbb{R}$ & 10 mg PO every 12 hours \\
\hline \multicolumn{2}{|l|}{ Rescue medication: } \\
\hline OxyNorm ${ }^{\circledR}$ Instant & 5 mg PO (maximum 6 times a day) \\
\hline \multicolumn{2}{|l|}{ Day 2 to day 5} \\
\hline Dafalgan $₫$ Forte & 1000 mg PO every 6 hours \\
\hline Vimovo ${ }^{2}$ & $500 \mathrm{mg}$ PO every 12 hours \\
\hline \multicolumn{2}{|l|}{ Rescue medication: } \\
\hline OxyContin $\AA$ & $10 \mathrm{mg}$ PO (maximum 2 times a day) \\
\hline OxyNorm ${ }^{\circledR}$ Instant & $\begin{array}{c}5 \mathrm{mg} \text { PO (maximum } 6 \text { times a day), } \\
\text { if Oxycontin is insufficient }\end{array}$ \\
\hline \multicolumn{2}{|l|}{ Day 6 to day 7} \\
\hline Dafalgan $\AA$ Forte & 1000 mg PO every 6 hours \\
\hline Vimovo® & $500 \mathrm{mg}$ PO every 12 hours \\
\hline \multicolumn{2}{|l|}{ After day $8 . .}$. \\
\hline Dafalgan $₫$ Forte & $\begin{array}{l}1000 \text { mg PO as needed } \\
\text { (maximum } 4 \text { times a day) }\end{array}$ \\
\hline
\end{tabular}

PO, by mouth ; IV, intravenous.

At $6: 30 \mathrm{pm}$, patients were seen by the surgeon and the anesthesiologist to approve the discharge. Discharge was permitted when patients were able to independently move from supine to standing and sitting to standing positions, ambulate for 30 meters, climb stairs, dress and perform bathroom transfers without help. Patients must exhibit stable vital signs and they were considered a candidate for home 
discharge after they demonstrated that they could tolerate a regular diet, that they were reasonably pain-free with the oral pain medications, and that they should have spontaneous urination. Only after all of the discharge criteria were met, were the patients given the option to make the final decision to leave the hospital or not. If patients were not feeling comfortable with the idea of going home, they could stay in hospital. Patients were instructed to reach the department at any time, in case of any potential complications relating to the hip replacement. Patients were encouraged to resume activities as tolerated and they were allowed to drive a car ten days after surgery. No home physiotherapy was prescribed.

Follow-up phone calls were made to each patient the first day after surgery by the surgeon and the anesthesiologist to ensure that recovery was progressing as expected. Patients were also seen at home by their general practitioner the first day after surgery for a blood sample and the checking of vital signs with a phone call thereafter by the surgeon to the general practitioner. Each patient was called again by the surgeon the third day after the surgery. The office personnel were instructed to record the contact with any study patient. All patients were seen post-operatively in the office after two weeks (the surgical suture was removed at this time), six weeks, three months, one year, and every five years thereafter.

All data were collected prospectively, but analyzed retrospectively. This study collected demographic information, including age, sex, BMI, reason for undergoing THA, pre-operative Harris Hip Score (HHS) (5) and ASA score. Primary clinical outcome measures included intra-operative complications, adverse events within the hospitalisation and the discharge home. During follow-up visits, patients were evaluated clinically and radiographically (after six weeks and after one year). Patients were asked about any complications they had experienced after surgery and less formally about the perception of their experience as outpatient and their satisfaction with their surgery and hospital stay, by asking the question, "would you recommend same-day THA to other?". Any contacts (whether it was by phone, fax, email or other means) to the surgeon's office, any early post-operative emergency department visits, acute office appointments or hospital re-admissions were registered and analysed.

The local ethics committee approved the study (B403201523492).

\section{RESULTS}

Between December 2015 and January 2018, a total of 260 elective primary THAs were performed and 52 of these $(20 \%)$ were enrolled for same-day discharge. Unilateral THA was performed on 49 patients, and three patients had bilateral THA in separate surgical episodes, resulting in a total of 52 THAs in 49 patients.

Of the 52 THAs enrolled in this study, 34 were men $(65,4 \%)$ and 18 were women $(34,6 \%)$. The average age at the time of surgery was 59,9 years (range, 43-76 years). 46 patients were between 50 and 75 years of age $(88,5 \%)$, and six patients were under 50 years of age $(11,5 \%)$. The average BMI was $26,2 \mathrm{~kg} / \mathrm{m}^{2}$ (range, 20,2-37,5 kg/m²). All operations were performed in an elective setting. The pre-operative diagnosis was osteoarthritis in 46 patients $(88,6 \%)$, avascular osteonecrosis in two patients $(3,8 \%)$, developmental dysplasia of the hip in one patient $(1,9 \%)$, radiation-induced coxarthritis in one patient $(1,9 \%)$, post-traumatic arthritis in one patient $(1,9 \%)$ and osteochondromatosis in one patient $(1,9 \%)$. The average pre-operative HHS was 66,11 points (range, 37-93 points). 18 patients were ASA I $(34,6 \%), 32$ patients were ASA II $(61,5 \%)$ and two patients were ASA III (3,9\%).

The post-operative follow-up period was at least three months (average, 10,9 months ; range, 3-26,8 months). No patients were lost to follow up.

Fifty-one $(98,1 \%)$ of 52 patients scheduled to receive a THA on an outpatient basis met the discharge criteria and achieved their goal of going to their own home on the day of surgery, and only one patient $(1,9 \%)$ required an overnight stay. This patient remained hospitalised due to neurological symptoms in the operated lower extremity with motor involvement with an inability to mobilise effectively, probably due to diffusion of the periarticular infiltration, which resolved during the first night. Then this patient met the discharge criteria on 
the following morning after an uneventful night and was discharged home at that time.

There were no intra-operative complications.

Five patients $(9,6 \%)$ had adverse events in the immediate post-operative setting. One patient $(1,9 \%)$ had orthostatic hypotension and two patients $(3,8 \%)$ presented a vasovagal reaction, with the consequence of a delay in physiotherapy sessions. However, all these patients responded to medication and were discharged on the day of surgery. One patient $(1,9 \%)$ had transient neurological symptoms in the operated lower extremity with motor involvement and with an inability to mobilise effectively, probably due to diffusion of the peri-articular infiltration. Neurological symptoms resolved within a few hours and the patient could complete his physiotherapy protocol and progressed to full weight bearing without any problems. One patient $(1,9 \%)$, who combined an orthostatic hypotension and transient neurological symptoms, required overnight observation. In that case too, neurological symptoms resolved within a few hours.

Two visits to the emergency room were recorded after the 52 procedures. There was one hospital readmission on the night of surgery for sanguineous drainage from the wound without any dehiscence or recurrence. The second patient required a visit to the emergency department 10 days after surgery for benign lower extremity swelling and wound drainage, which was the result of seroma formation, without re-admission. No surgery was performed in these cases, and the wounds healed otherwise without any further complications.

Seven acute office appointments were recorded during the first three months after surgery. One patient came 10 days post-operatively for a broken surgical suture into the wound (because he tried to remove it himself). Two patients came after one month post-operatively for abnormal recurrence of pain. For one, it was an iliopsoas tendinitis while a non-displaced periprosthetic fracture without history of trauma was discovered for the second one. One patient came three times (after four, five and eight weeks post-operatively) for pain due to a distal migration of the femoral stem, and one patient had no problem but came to change the bandage.
One patient $(1,9 \%)$ presented a sanguineous drainage from the wound, required an emergency room visit and was re-admitted as said before. Two patients $(3,8 \%)$ had a broken surgical suture into the wound (one patient tried to remove it himself), with no further complication. Benign lower extremity swelling developed 10 days after one procedure $(1,9 \%)$. One patient $(1,9 \%)$ combined a wound drainage and a benign lower extremity swelling and required an emergency room visit 10 days after the procedure, without re-admission. No surgery was performed and the wounds healed otherwise without any further complications. There was no wound necrosis or infections in any of the 52 patients.

Two patients $(3,8 \%)$ presented an iliopsoas tendinitis respectively, after four weeks and after three months post-operatively while two other patients $(3,8 \%)$ presented a trochanteritis after one month and after six weeks post-operatively. All these patients were treated with anti-inflammatories, painkillers and application of ice without further complication or recurrence.

Two patients $(3,8 \%)$ sustained a non-displaced periprosthetic fracture without history of trauma which didn't require any revision surgery and was treated by partial weight bearing, using two crutches for six weeks. One patient $(1,9 \%)$ presented a distal migration of the femoral stem without repercussion on mobility. No patient experienced a hip dislocation. All patients had well-fixed prostheses with no progressive radiolucencies and no other signs of prosthetic loosening at the latest follow-up.

One patient $(1,9 \%)$ presented a superficial venous thrombosis after six weeks post-operatively and was

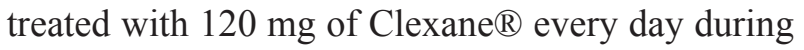
three months.

HHS increased from 66,11 points (range, 3793 points) pre-operatively to 79,63 points (range, 37-100 points) at two weeks post-operatively, to 91,13 points (range, 49-100 points) at six weeks post-operatively and to 99,44 points (range, 95-100 points) at three months post-operatively.

When questioned on the perception of their experience as outpatient, 51 patients $(98,1 \%)$ were satisfied they were able to go home the day of surgery and would recommend same-day THA to others. Only one patient $(1,9 \%)$ reported a signi- 
ficantly negative experience. This patient (who didn't present any post-operative complication) believed he would have been better served having stayed in hospital, at least overnight.

\section{DISCUSSION}

It has been described that strict criteria should be met before considering a patient for outpatient THA (3). Outpatient surgery is not for everyone. According to Gromov et al (6), outpatient THA is feasible in an unselected patient population in about $15-20 \%$ of patients. This is in accordance with our rate of $20 \%$.

One of the most important concerns with outpatient surgery has been the potential for increased re-admissions to the hospital and visits to emergency departments. In our study, there were two visits to the emergency department, one re-admission and seven acute office appointments during the first three months after surgery and our staff didn't notice any increase in patient phone calls, fax or emails, in comparison to those received from our hospital based patients.

Pain management was viewed as a primary challenge to outpatient THA. The combination of a multimodal pain management protocol including the use of a periarticular injection during surgery effectively treated the post-operative pain and prevented any hospitalisations for pain control. In our study, pain was not a substantial problem with regard to discharge on the day of surgery, demonstrating well-controlled pain with our protocol in place (Table I).

Regarding the (pulmonary embolism) PE risk, standard procedure for THA presents a risk for PE till 90 days after a THA (7). Therefore, the PE risk difference between inpatient and outpatient procedures is just about the few first days. A Risk Stratification System for Pulmonary Embolism After Elective Primary Total Joint Arthroplasty has been suggested (7) and should allow to identify the patients more at risk and prevent such a complication in an outpatient setting. Rapid mobilisation and reduced inflammatory response in anterior approaches are also factors susceptible to prevent part of the complications (8) as a relationship is observed between inflammatory response and estimated complication rate (9).

In the present study, 51 of the 52 selected patients $(98,1 \%)$ who were scheduled to receive THA on an outpatient basis, went home on the day of surgery and no patient was discharged to a skilled nursing or inpatient rehabilitation facility. This is comparable with the discharge rates reported in the literature $(10,11,12,13,14,15)$.

In our study, five patients $(9,6 \%)$ presented immediate complications and 13 patients $(25 \%)$ presented early three-month post-operative complications. There were no medical events that put the patients at risk by having surgery performed on an outpatient basis, suggesting that appropriately selected patients are safe to undergo outpatient THA. This is in accordance with the results of two meta-analysis $(16,17)$ of the published literature, suggesting that outpatient THA can be performed safely and effectively for carefully selected patients.

Our study demonstrated improvements in HHS from pre-operatively to three months postoperatively: HHS increased from 66,11 points to 99,44 points. Moreover, 51 patients $(98,1 \%)$ were satisfied with the post-operative results while explaining how they functioned in their daily life.

There are several limitations to our study. Potential limitations of this study include the relatively small sample size $(n=52)$. As we know that the incidence of PE after THA is one per 250 (18), we didn't face this major complication. Secondly, all the operations were performed by a single surgeon $(\mathrm{EW})$, at the same center. A third limitation is the lack of a control group in a non-randomised noncomparative design, making it difficult to establish conclusions, but study questions were about safety and efficacy, not attempting to prove that a sameday discharge was better than a longer hospital stay. Then, our study is limited by a significant inherent selection bias associated with the highly selected patient population. This stringent patient selection likely contributes to the high rate of discharge on the day of surgery, and the low re-admission rate. Furthermore, some patients came specifically to this clinic to be operated by this surgeon because they did know that he was doing outpatient THA in his practice. The fifth limitation of the study is that 
the follow-up was only three months. And finally, limitations exist with the patient satisfaction survey. There are no validated questionnaires.

With this first Belgian experience reporting on outpatient primary THA, our data demonstrate that early discharge does not result in excessive re-admissions or other post-discharge complications due to same-day discharge. This study suggests that THA can be performed safely and successfully for appropriately selected and motivated patients without requiring additional work for the surgeon's office, and that patients who experienced that ambulatory protocol declared themselves highly satisfied. In order to perform outpatient THA, an accelerated clinical pathway with a multidisciplinary approach involving a range of health-care professionals must be developed and implemented. Moreover, preoperative patient counseling is crucial : patients must be given appropriate goals and expectations through pre-operative education. To definitively elucidate the safety and feasibility of outpatient THA, further studies comparing inpatient and outpatient THA outcomes between randomised cohorts of patients under similar operating circumstances should be done. Finally, further cost analysis should investigate the true financial benefits of outpatient THA treatment.

\section{REFERENCES}

1. Kurtz SM, Lau E, Ong K, Zhao K, Kelly M, Bozic KJ. Future young patient demand for primary and revision joint replacement : national projections from 2010 to 2030. Clin Orthop Relat Res. 2009 ; 467(10) : 2606-12.

2. Stowers MD, Manuopangai L, Hill AG, Gray JR, Coleman B, Munro JT. Enhanced recovery after surgery in elective hip and knee arthroplasty reduces length of hospital stay. ANZ J Surg. 2016 ; 86(6) : 475-9.

3. Argenson JN, Husted H, Lombardi A Jr, Booth RE, Thienpont E. Global Forum : An international perspective on outpatient surgical procedures for adult hip and knee reconstruction. J Bone Joint Surg Am. 2016 ; 98(13) : e55.

4. Hackett NJ, De Oliveira GS, Jain UK, Kim JY. ASA class is a reliable independent predictor of medical complications and mortality following surgery. Int J Surg. 2015 ; 18 : 18490.

5. Nilsdotter A, Bremander A. Measure of hip function and symptoms: Harris hip score (HHS), hip disability and osteoarthritis outcome score (HOOS), Oxford hip score (OHS), Lequesne index of severity for osteoarthritis of the hip (LISOH), and American Academy of Orthopedic Surgeons (AAOS) hip and knee questionnaire. Arthritis Care Res (Hoboken). 2011 ; 63(Supp111) : S200-7.

6. Gromov K, Kjaersgaard-Andersen P, Revald P, Kehlet H, Husted H. Feasibility of outpatient total hip and knee arthroplasty in unselected patients. Acta Orthop. 2017 ; $88(5)$ : 516-21.

7. Bohl DD, Maltenfort MG, Huang R, Parvizi J, Lieberman JR, Della Valle CJ. Development and validation of a risk stratification system for pulmonary embolism after elective primary total joint arthroplasty. $J$ Arthroplasty. 2016 ; 31(9 Suppl) : 187-91.

8. Bergin PF, Doppelt JD, Kephart CJ, Benke MT, Graeter JH, Holmes AS. et al. Comparison of minimally invasive dirct anterior versus posterior total hip arthroplasty based on inflammation and muscle damage markers. J Bone Joint Surg Am. 2011 ; 93(15) : 1392-98.

9. Chen X, Wang T, Li J, Kang H. Relationship between inflammatory response and estimated complication rate after total hip arthroplasty. Chin Med J (Engl). 2016 ; 129(21) : 2546-51.

10. Klein GR, Posner JM, Levine HB, Hartzband MA. Same day total hip arthroplasty performed at an ambulatory surgical center : 90 -day complication rate on 549 patients. $J$ Arthroplasty. 2017 ; 32(4) : 1103-6.

11. Parcells BW, Giacobbe D, Macknet D, Smith A, Schottenfeld M, Harwood DA, et al. Total joint arthroplasty in a stand-alone ambulatory surgical center : shortterm outcomes. Orthopedics. 2016 ; 39(4) : 223-8.

12. Toy PC, Fournier MN, Throckmorton TW, Mihalko WM. Low rates of adverse events following ambulatory outpatient total hip arthroplasty at a free-standing surgery center. J Arthroplasty. 2018 ; 33(1) : 46-50.

13. Dorr LD, Thomas DJ, Zhu J, Dastane M, Chao L, Long WT. Outpatient total hip arthroplasty. J Arthroplasty. 2010 ; 25(4) : 501-6.

14. Berger RA, Sanders SA, Thill ES, Sporer SM, Della Valle C. Newer anesthesia and rehabilitation protocols enable outpatient hip replacement in selected patients. Clin Orthop Relat Res. 2009 ; 467(6) : 1424-30.

15. Berend KR, Lombardi AV Jr, Berend ME, Adams JB, Morris MJ. The outpatient total hip arthroplasty : a paradigm change. Bone Joint J. 2018 ; 100-B(1 Suppl A) : 31-5.

16. Hoffmann JD, Kusnezov NA, Dunn JC, Zarkadis NJ, Goodman GP, Berger RA. The shift to same-day outpatient joint arthroplasty: a systematic review. J Arthroplasty. 2018 ; 33(4) : 1265-74.

17. Pollock M, Somerville L, Firth A, Lanting B. Outpatient total hip arthroplasty, total knee arthroplasty, and unicompartimental knee arthroplasty : a systematic review of the literature. JBJS Rev. 2016 ; 4(12) : e4.

18. Memtsoudis SG, Besculides MC, Gaber L, Liu S, Gonzalez Della Valle A. Risk factors for pulmonary embolism after hip and knee arthroplasty : a populationbased study. Int Orthop. 2009 ; 33(6) : 1739-45. 